\title{
Seminare 2006
}

\section{Praxiseröffnung/-übernahme}

Themen (Details $\rightarrow$ www.fmhservices.ch) Juristische Aspekte - Ehe- und Erbrecht - Praxiseinrichtung - Praxisadministration - Unternehmensbewertung einer Arztpraxis - Finanzierung einer Arztpraxis - Versicherungen.

\section{Sponsoren}

Versicherung der Schweizer Ärzte, Schweizerische Ärzte-Krankenkasse, Druckerei Schmid-Mogelsberg, Labornetz daCapo, Unilabs SA, Labor medica, Kern Concept AG und EMH Schweizerischer Ärzteverlag AG

\section{Datum}

$\begin{array}{lll}\text { Nr. } 06 \text { Donnerstag, 9. November } 2006 & \text { Kantons- } \\ \text { Basel } & 9.00-16.30 \mathrm{Uhr} & \text { spital }\end{array}$

\section{Buchhaltung und Steuern}

\section{Themen (Details $\rightarrow$ www.fmhservices.ch)}

Buchführung (Massnahmen vor Praxiseröffnung/-übernahme, Standardkontenplan, doppelte Buchhaltung, EDV-unterstützte Buchführungslösung), Steuern (Steuern als Selbständigerwerbender, Steuerdomizil Privat/Geschäft, strategische und operative Steuerplanung, Steueroptimierungsmöglichkeiten).

\section{Sponsor}

EMH Schweizerischer Ärzteverlag

\section{Datum}

Nr. 16 Donnerstag, 23. November 2006 KantonsBasel 9.30-16.30 Uhr spital

\section{Praxiscomputerworkshop}

\section{Inhalt}

Präsentation von sechs Praxisadministrationssoftwarelösungen (Leistungserfassung, elektronisches Abrechnen unter Einbezug der TrustCenter, Agendaführung, elektronische Krankengeschichte, Statistiken, Laborgeräteeinbindung, Finanzbuchhaltungslösungen usw.).

\section{Ziel}

Die Workshopteilnehmer/innen erhalten einen ersten Überblick über führende Softwarelösungen. Sie erfahren, wie bei der Evaluation eines
Ärztepaketes zweckmässigerweise vorgegangen wird und welche Fehler vermieden werden sollten.

\section{Datum}

$\begin{array}{lll}\text { Nr. } 19 & \begin{array}{l}\text { Donnerstag, 30. November } 2006 \\ \text { Zürich }\end{array} & \begin{array}{l}\text { Hotel } \\ \text { 14.00-17.30 Uhr }\end{array} \\ & & \begin{array}{l}\text { Inter- } \\ \text { continental }\end{array}\end{array}$

\section{Folgende Softwareanbieter können Sie kennenlernen}

\section{Ärztekasse, Urdorf (MediWin CB Pro 6)}

Als Marktleader seit 1964 im Bereich Praxisadministration können Sie bei der Ärztekasse auf eine ganzheitliche Lösung für Ihre Fragen betreffend Abrechnungsvarianten, Computerwahl, Netzwerke, Formular- und Briefbearbeitung usw. zählen. Die statistischen Erhebungen (im Auftrag der FMH, Roko) sind ein anerkanntes betriebswirtschaftliches und standesorganisatorisches Hilfsmittel für die moderne Praxisführung. Nebst innovativen Ideen und Lösungen sowie Kooperationspartnerschaft mit 10 TrustCentern ist uns eine umfassende Kundenbetreuung ein Anliegen. Unsere Standorte befinden sich in Basel, Bern, Chur, Crissier, Genf, Le Landeron, Lugano, Luzern, St. Gallen, Thônex und Zürich. Sie definieren Ihre Wünsche - wir bieten Ihnen die Lösung!

\section{Delemed AG, Kehrsatz (delemed ${ }^{\circledR}$ PEX II)}

Delemed AG entwickelt und vertreibt seit 15 Jahren erfolgreich PEX II, die einfache, sympathische und effiziente Software für die Arztpraxis und Kliniken. Durch einen modularen Aufbau kann das System jederzeit den aktuellen Bedürfnissen des Kunden angepasst werden. Dank unserer Vielseitigkeit im medizinischen Umfeld und unserem Kundenstamm von über 650 Anwendern sind wir ein optimaler Partner für Sie.

\section{Kern Concept AG, Gossau (AESKULAP)}

AESKULAP ist ein extrem schnelles Windowsprogramm, das zusammen mit einer SQL-Datenbank höchste Verarbeitungsgeschwindigkeit, Kompaktheit und schnellste Zugriffsmöglichkeiten gewährleistet. AESKULAP bietet für alle Bedürfnisse eine optimale Lösung: vom einfachen Abrechnungssystem mit sämtlichen Leistungserfassungsmöglichkeiten bis zur vollstrukturierten elektronischen KG-Führung, die in der Praxis keine Wünsche offenlässt. Rund 130 Praxen 
arbeiten bereits mit der elektronischen KG-Führung; ohne Papierarchivierung. AESKULAP ist ein modular aufgebautes, anpassbares System mit einer Vielzahl von Modulen. Auch verfügt es über einen integrierten TARMED-Validator sowie einen TARMED-Optimizer. Das gewährleistet vollständige Rechnungen, da technische Grundleistungen, Prozentzuschläge und Alterszuschläge vollautomatisch hinzugefügt werden, ohne dass dafür unzählige Leistungsblöcke notwendig sind.

\section{Triamun AG, Gümligen}

(PRAXIDATA, TRIAMUN)

Wir entwickeln und vermarkten innovative Softwarelösungen für das Praxis- und Apothekenmanagement. Individuelle Beratung, die gesamte Soft- und Hardware, einen umfassenden Support und die Schulung erhalten Sie von uns aus einer Hand. Unsere Produkte und Dienstleistungen auf den beiden Plattformen ASP (triamun ${ }^{\circledR}$ ) und .NET (Praxidata) bieten für jedes Bedürfnis die geeignete Lösung. Die Triamun-Softwarelösungen auf der ASP-Plattform, für Apotheken und für die Arztpraxis, basieren auf einer innovativen browsergestützten Technologie und wurden gemeinsam mit Ärzten und Apothekern entwickelt. So sind intuitiv bedienbare Managementlösungen für Apotheken und Praxen entstanden, die dem Bedürfnis nach zentralem Outsourcing Rechnung tragen. Die Praxidata-Softwarelösung auf der .NET-Plattform, mit seinen Zusatzmodulen für die Arztpraxis, wurde während 20 Jahren den Kundenbedürfnissen ständig angepasst, so dass sie heute als modernste, lokale Client/ Server-Lösungen optimal auf die Abläufe in der Arztpraxis zugeschnitten ist.

\section{TMR - Triangle Micro Research AG, Hölstein (WinMed ${ }^{\circledR}$ )}

Die TMR AG wurde von Ärzten, Wissenschaftlern, Unternehmern und Dozenten der Uni Basel 1993 als «Spin-Off»-Firma gegründet. Den Schwerpunkt in der Entwicklung der TMR AG bilden Anwendungen im Bereich der medizinischen Telekommunikation, Internet sowie der mehrmandanten- und mehrplatzfähigen Ärztesoftware WinMed ${ }^{\circledR}$. WinMed ${ }^{\circledR}$ ist ein vollständiges Administrationssystem mit integrierter Kommunikationsplattform, Bild- und Dokumentenverwaltung für Arztpraxen. WinMed ${ }^{\circledR}$ wird nach ausgereifter mehrjähriger Pilotphase erfolgreich seit 1998 verkauft und zählt heute zu den meistgekauften Ärztesoftwarepaketen. Vertrieben wird WinMed $^{\circledR}$ von der TMR AG selbst, im Tessin von GFP Mediconsul in Massagno.

\section{Vitodata AG, Ohringen b. Winterthur (vitomed Administrationssystem)}

Die Vitodata AG feierte das letzte Jahr das 25jährige Bestehen. Die Unternehmung konzentriert sich auf Praxis- und Kliniklösungen. Die innovative Haltung eröffnet laufend neue Einsatzgebiete - immer mit dem entsprechenden Nutzen für die Anwender. In den ersten 25 Jahren stand die Abrechnung mit der EDV im Vordergrund. Heute ist die Vitodata AG auch bei der elektronischen Krankengeschichtenführung an der Spitze im Schweizer Markt. Die Marktführerschaft ist für das ganze Team der Vitodata AG eine Verpflichtung, im Sinne des Investitionsschutzes für die Kunden den Fortbestand zu sichern und unternehmerisch und ethisch stets korrekt $\mathrm{zu}$ handeln.

\section{Anmeldung und Auskunft}

FMH Consulting Services, Claudine Tannari, Burghöhe 1, 6208 Oberkirch, Tel. 04192500 77, Fax 0419210586 oder via Internet unter www.fmhservices.ch.

\section{Hinweis}

Bei sämtlichen Seminaren, bei welchen die Kosten teilweise oder gänzlich von Seminarsponsoren gedeckt werden, werden die Teilnehmeradressen den jeweiligen Sponsoren zur Verfügung gestellt.

\section{Annullierungsbedingungen}

Bei den Gratisseminaren werden bei Abmeldungen oder Fernbleiben folgende Unkostenbeiträge erhoben:

- Fr. 50.- pro Person ab 14 Tage vor Seminarbeginn;

- Fr. 80.- pro Person ab 7 Tage vor Seminarbeginn oder Fernbleiben.

Bei Abmeldungen oder Fernbleiben bei kostenpflichtigen Seminaren werden die folgenden Unkostenbeiträge erhoben:

- 30\% der Seminarkosten ab 14 Tage vor Seminarbeginn;

- $100 \%$ der Seminarkosten ab 7 Tage vor Seminarbeginn oder Fernbleiben.

\section{Seminarsponsoren 2006}

Im November beginnt die letzte Seminarreihe 2006 der FMH Consulting Services. Die Unterstützung von verschiedenen Sponsoren ermöglicht es, diese Seminare für FMH-Mitglieder teils kostenlos, teils kostengünstig anzubieten. Gerne stellen wir Ihnen diese Firmen in einem Kurzporträt vor. 


\section{dacapo}

E-Mail: info@dacapo.ch

Website: www.dacapo.ch

daCapo ist eine lose Interessengemeinschaft von fünf eigenständigen medizinischen Labors, die mit Arztpraxen eine sehr persönliche und unkomplizierte, von Sicherheit, Qualität und Effizienz geprägte Zusammenarbeit pflegen. Bei uns stehen Sie immer in direktem, kollegialem Kontakt mit den Laborinhabern und den leitenden Mitarbeiterinnen und Mitarbeitern, die Ihre Analyseaufträge von A bis Z persönlich betreuen. Med. Labor Olten Dr. Vögeli (www. mlo.ch) - Rothen Medizinische Laboratorien, Basel (www.labor-rothen.ch) - Mikrogen AG, Zürich (www.mikrogen.ch) • Labor Prof. Krech und Partner AG, Kreuzlingen (www.labor.ch) • Medics Labor AG, Bern (www.medics-labor.ch)

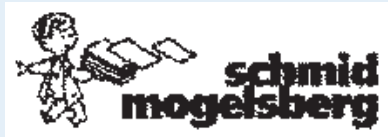

P. Schmid + Co. AG, Ärztedrucksachen Sonnmattstrasse 1, 9122 Mogelsberg Tel. 07137560 80, Fax 0713756081 E-Mail: schmid.mogelsberg@bluewin.ch Website: www.schmid-mogelsberg.ch

\section{Seit 70 Jahren auf Ärztedrucksachen spezialisiert!}

Bei der Gestaltung von zweckmässigen Arztformularen (Patientenkarten, Labor- und Statusformulare usw.) profitieren Sie von der langjährigen Erfahrung. Die Vergangenheit hat gezeigt, dass sich kaum zwei Ärzte für den gleichen Druck entscheiden. Zweckmässige Materialauswahl, einwandfreie Verarbeitung, freundliche und kompetente Beratung, schnelle Lieferung und die Ausführung von Spezialwünschen diese Dienstleistungen schaffen die Grundlage zu einem langjährigen Vertrauensverhältnis. Auf Wunsch wird jeweils eine individuell auf die Fachrichtung zusammengestellte Druckmusterkollektion den Ärztekunden zugestellt.

An über 9000 Ärzte werden ausserdem viele Artikel wie Briefpapier, Couverts, Visitenkarten, EDV-Formulare, Röntgenfilmtaschen, hygienische Papiere und Büromaterial für die tägliche Administration in der Arztpraxis geliefert. Neu werden die praktischen TARMED-Formulare angeboten.

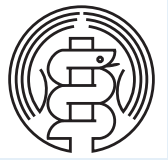

Schweizerische Ärzte-Krankenkasse Oberer Graben 37, 9001 St. Gallen

Tel. 07122718 18, Fax 0712271828

E-Mail: info@saekk.ch

Website: www.saekk.ch

Die richtige Adresse für Erwerbsausfalldeckungen, Kollektivkrankenkasse und Versicherungsplanung

Mit mehr als 100 Jahren Erfahrung kennt unsere Organisation auch heute die Bedürfnisse der Ärztinnen und Ärzte. Sie bietet entsprechend durchdachte und kostengünstige Lösungen an, sowohl für Praxiseröffner/innen wie auch für selbständige und angestellte Ärztinnen und Ärzte.

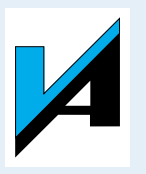

Versicherung der Schweizer Ärzte

Länggassstrasse 8, 3000 Bern 9

Tel. 03130125 55, Fax 0313025156

E-Mail: versa@versa.ch

Website: www.versa.ch

Spezialisiert auf die Bedürfnisse von Ärztinnen und Ärzten und deren Ehegatten bietet die Versicherung der Schweizer Ärzte individuelle, den jeweiligen Bedürfnissen angepasste Versicherungslösungen im Bereich der privaten Vorsorge an.

\section{() Unilabs}

Website: www.unilabs.ch

\section{UNILABS - Ihr Schweizer Qualitätslabor} schnell, zuverlässig und nah

Unilabs ist im Bereich der medizinischen Analysen ein kompetenter, transparenter und zuverlässiger Partner. Wir bieten Ihnen überall in der Schweiz ein komplettes Analysenspektrum, umfassende Dienstleistungen und kompetente Fachberatung an. In der Deutschschweiz sind dies die Medizinische Analytik AG, MAB, das Labor Brunnhof und das Zytologielabor Dr. Denzler in der Region Bern und Mittelland, die Labors Diagnostica AG in Zürich und das Enzymlabor Dr. H. Weber AG in St. Gallen. 
Unilabs bietet nicht nur medizinische Laboranalysen, sondern auch wertvolle individuelle Zusatzdienstleistungen wie Beratung und Weiterbildung für alle Mitglieder des Praxisteams, Unterstützung bei der nachhaltigen Reduktion des Praxisaufwandes und Beratung bei Praxisund Laborbedarf.

Die Unilabs-Strategie zielt darauf ab, ihre vielfältigen und regionalen Dienstleistungen über eine fundierte wissenschaftliche Struktur sicherzustellen. Zur Gewährleistung einer hochstehenden Qualität werden stets die notwendigen Investitionen für Ausrüstung und Ausbildung getätigt.

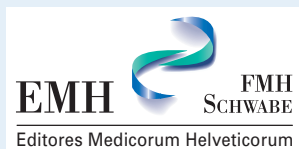

EMH Schweizerischer Ärzteverlag AG

Farnsburgerstrasse 8, 4132 Muttenz

Tel. 06146785 55, Fax 0614678556

E-Mail: verlag@emh.ch

Website: www.emh.ch

\section{EMH, der Verlag der Ärztinnen und Ärzte}

Der Verlag EMH Schweizerischer Ärzteverlag AG wurde 1997 gegründet. EMH ist eine Kooperation zwischen der Verbindung der Schweizer Ärztinnen und Ärzte FMH und dem Basler Verlagshaus Schwabe AG.

Hauptpublikationen von EMH sind die Zeitschriften «Schweizerische Ärztezeitung», das offizielle Publikationsorgan der FMH, das «Swiss Medical Forum» mit praxisorientierten Fortbildungsbeiträgen, «Swiss Medical Weekly», die Plattform für klinisch orientierte Wissenschaftler, sowie «PrimaryCare», offizielles Organ des Kollegiums für Hausarztmedizin (KHM) und der Schweiz. Gesellschaft für Allgemeinmedizin (SGAM).

Als erfolgreiches Onlineangebot darf unter anderem die Fortbildung (www.smf-cme.ch) des Swiss Medical Forum bezeichnet werden. Steigende Zugriffszahlen und die Akkreditierung durch die Fachgesellschaften SGAM und SGIM als strukturierte und nachweisbare Fortbildung belegen diesen Erfolg.

Weitere Projekte und Dienstleistungen runden das umfangreiche Verlagsangebot $\mathrm{ab}$.

\section{medica}

MEDIZINISCHE LABORATORIEN Dr. F. KAEPPELI AG

MEDIZINISCHE LABORATORIEN

DR. F. KAEPPELI AG

Eidgenössisch anerkannte Laboratorien
Wolfbachstrasse 17, 8024 Zürich

Tel. 04426999 99, Fax 0442699909

E-Mail: info@medica-labor.ch

Website: www.medica-labor.ch

Der promovierte Mikrobiologe und Biochemiker Dr. F. Käppeli, Laborspezialist FAMH, übernahm 1976 das heute fast 50jährige Unternehmen und gründete als dessen Leiter und Inhaber die Einzelfirma medica. Der wichtigste unternehmerische Leitgedanke von Dr. F. Käppeli heisst kontinuierliche Innovation und Schaffung wegweisender Standards auf allen Gebieten der Labormedizin: Mikrobiologie inklusive Parasitologie, Serologie, Immunologie, klinische Chemie, Hämatologie, molekulare Diagnostik und Pathologie in Human- und Veterinärmedizin. So entstand ein Kompetenzzentrum für Labordiagnostik von gesamtschweizerisch einmaliger Fachkapazität. Die modernst ausgebauten Laboratorien werden laufend erweitert und befinden sich im Herzen von Zürich. Gegen 200 Angestellte der Partnerlabors, begleitet von Spezialisten aus Medizin, Pharmakologie, Naturwissenschaften und Technik, garantieren höchste Professionalität.

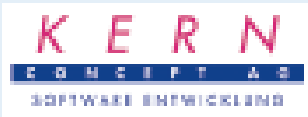

Kern Concept AG

Wilerstrasse 75, 9200 Gossau

Tel. 07138880 30, Fax 0713888031

E-Mail: info@kernconcept.ch

Website: www.kernconcept.ch

KERN CONCEPT AG entwickelte und betreut das Administrationsprogramm AESKULAP, das heute in rund 1200 Arztpraxen erfolgreich eingesetzt wird. AESKULAP ist ein extrem schnelles Windowsprogramm, das zusammen mit einer SQL-Datenbank höchste Verarbeitungsgeschwindigkeit, Kompaktheit und schnellste Zugriffsmöglichkeiten gewährleistet. AESKULAP bietet für alle Bedürfnisse eine optimale Lösung: vom einfachen Abrechnungssystem mit sämtlichen Leistungserfassungsmöglichkeiten bis zur vollstrukturierten elektronischen KG-Führung, die in der Praxis keine Wünsche offenlässt. AESKULAP ist ein modular aufgebautes, anpassbares System mit einer Vielzahl von Modulen. Auch verfügt es über einen integrierten TARMEDValidator sowie einen TARMED-Optimizer. Das gewährleistet vollständige Rechnungen, da technische Grundleistungen, Prozentzuschläge und Alterszuschläge vollautomatisch hinzugefügt werden. 